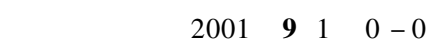

Biodiversity Science

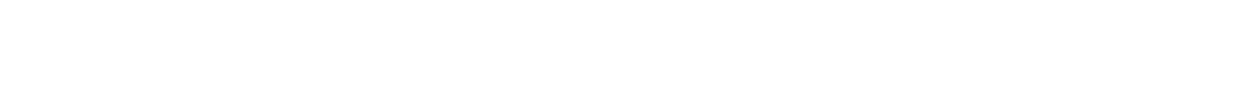

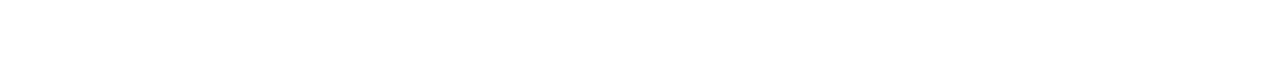

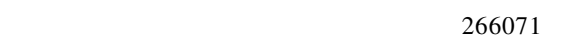

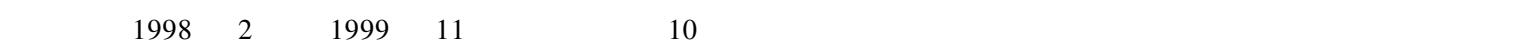

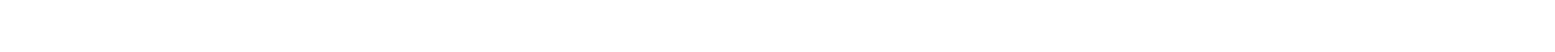

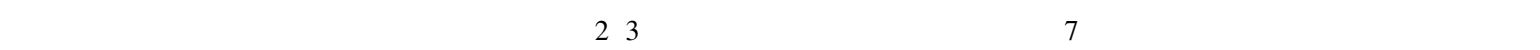

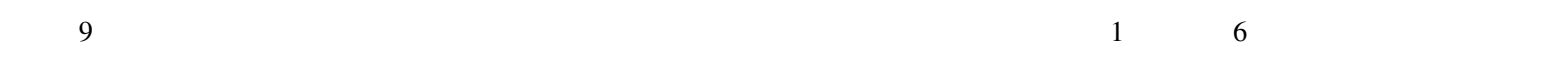

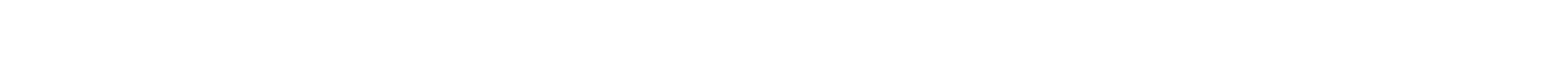

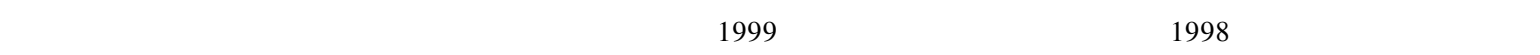

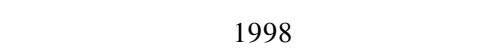

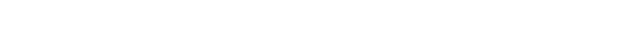

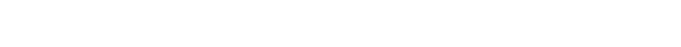

\section{Study on the species diversity of macrobenthic fauna in Jiaozhou Bay}

LI Xin - zhengf-YU Hai - Yanf-W ANG Yong - Qiangf-SHUAI Lian - Meif-ZHANG Bao - Linfand LIU Rui - Yu

Institute of Oceanology $f-$ - $h$ hinese Academy of Sciencesf-@ingdao 266071

\begin{abstract}
O}^{\circ}$ Field research of macrobenthic fauna at 10 stations in Jiaozhou Bay was carried out seasonally from February of 1998 to November of 1999. There were fewer species and the indices of biodiversity f" Shannon - Wiener index $f$ Gere lower in Stations $2 £ \mathcal{f} f-7$ and 9 than in other stations. Stations 2 and 3 were situated in the inner area near Cangkouf $\mathbf{a}$ heavily polluted area. Stations 7 and 9 were situated at the mouth of the bayfwhere the bottom sediment was coarse sand. The indices in Stations 1 and $6 f$ where the bottom sediment was soft mudf-were high. The average Shannon - Wiener index of all the 10 stations was lower in 1999 than in $1998 \mathrm{f}$ although the difference was not remarkable. In both of the two yearsf $\neg$ the average Shannon - Wiener indices were lower in spring and autumn than in winter and summerf» Pielouj $\bar{s}$ evenness index was highest in summer and lowest in spring $f$ »and the species richness index was slightly higher in winter and slightly lower in autumn. The relationship between biodiversity of the macrobenthos in Jiaozhou Bay and human activities should be studied further.
\end{abstract}

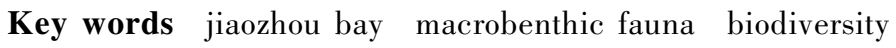

\section{1 Ò̃Ñ̂̃}

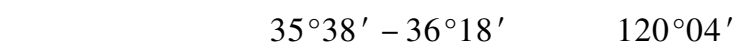

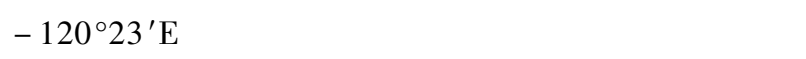

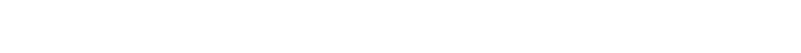

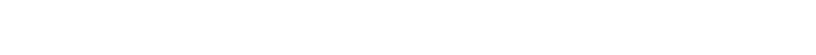

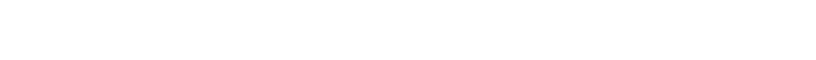

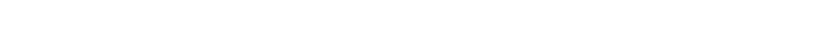

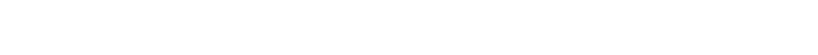

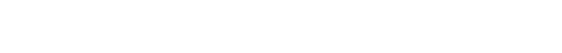

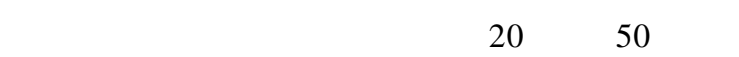




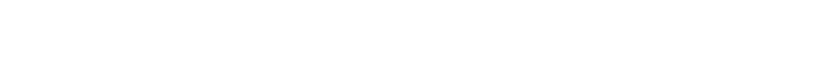

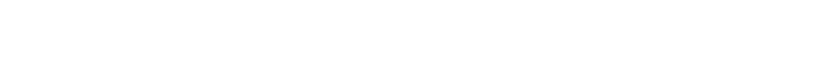

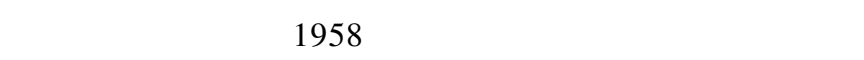

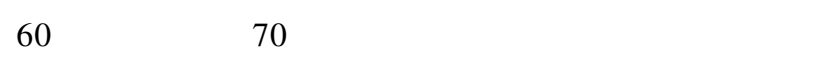

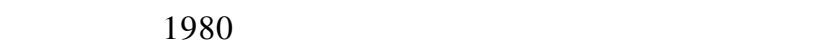

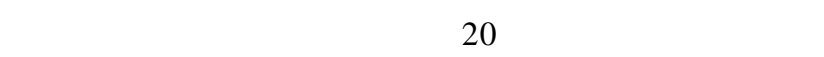

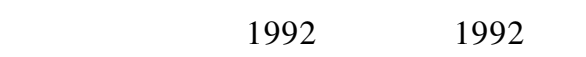

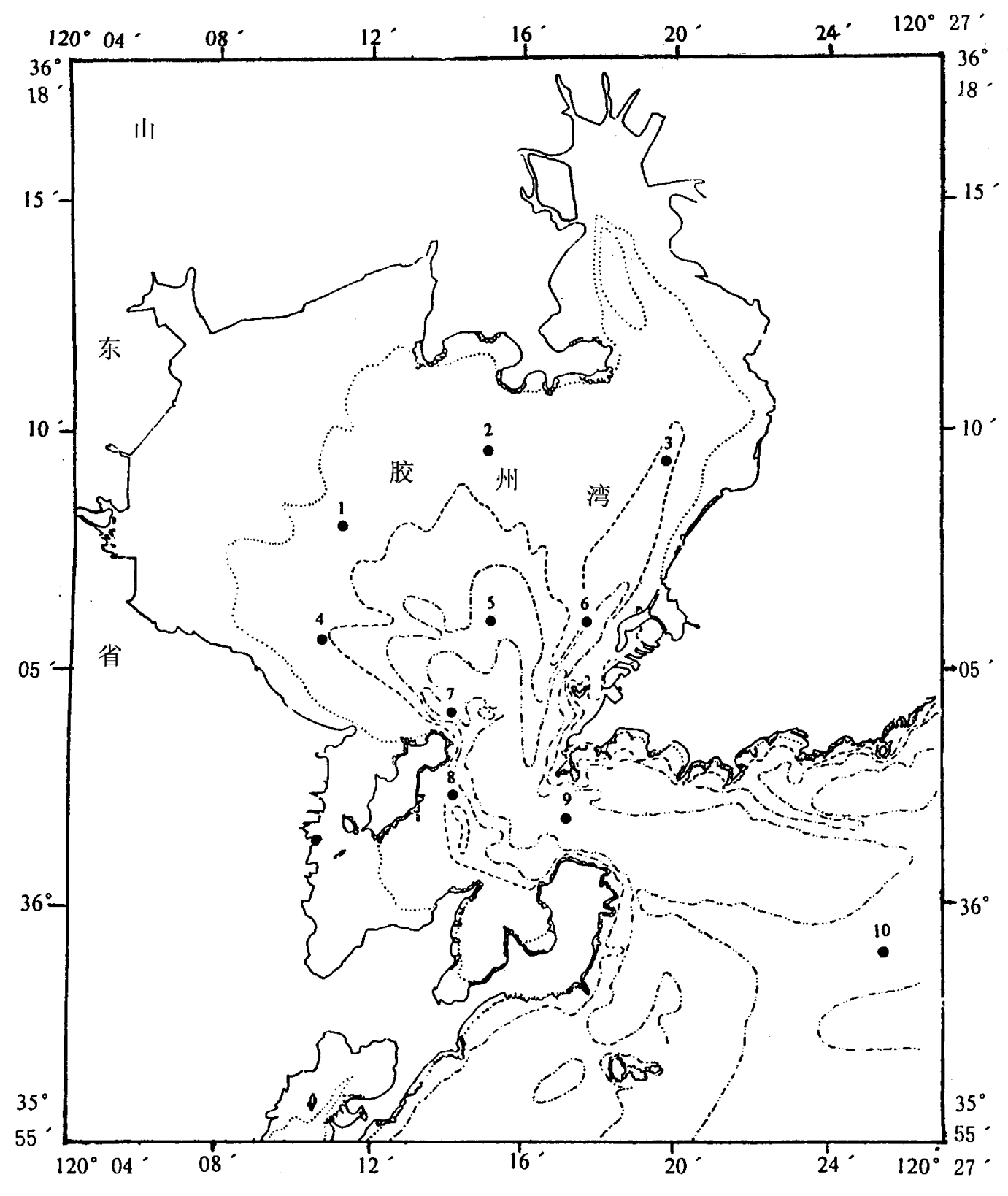

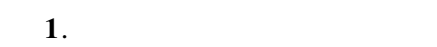

Fig. 1. The distribution of sampling stations during monitoring period.

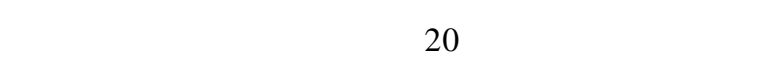

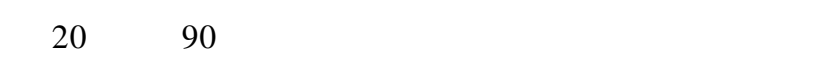

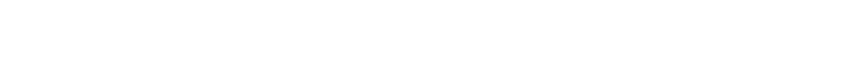

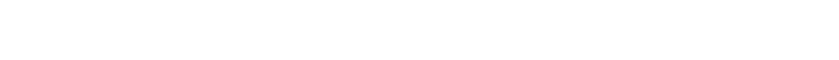

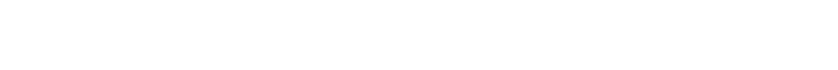

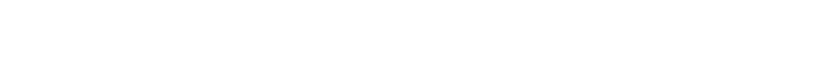

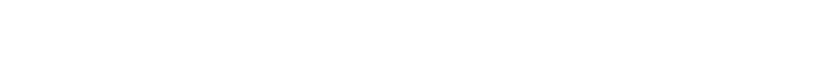

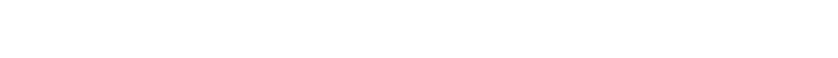




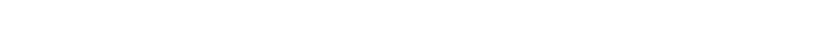

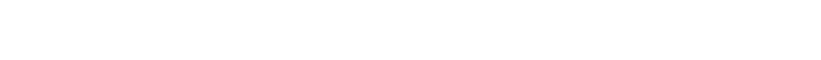
XÊÄ i f

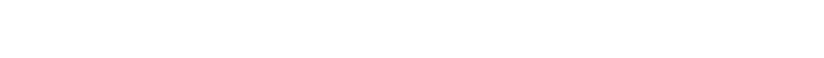

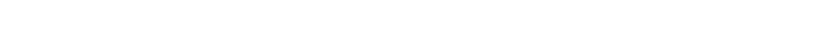

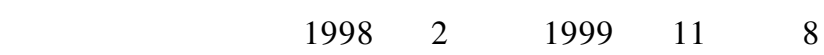

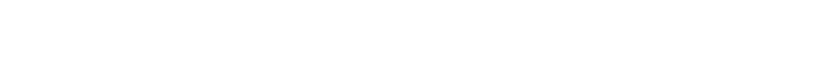

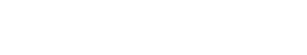

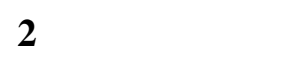

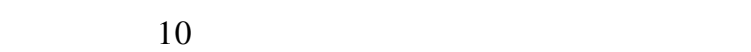

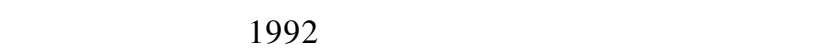

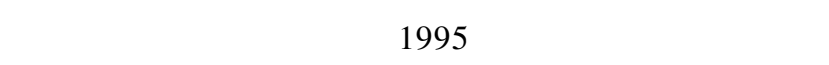

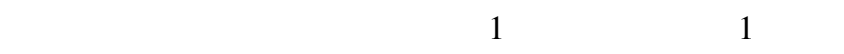

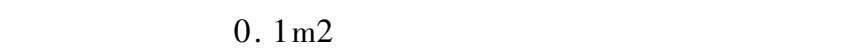

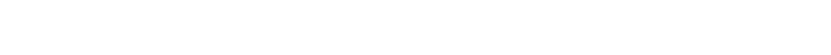

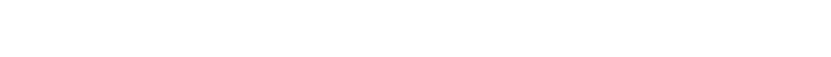

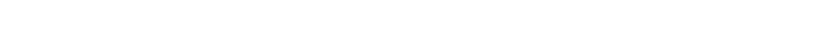

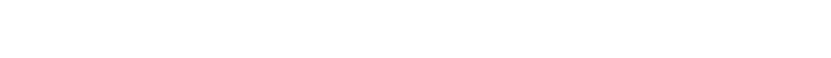
๒® Ö ö; $€$

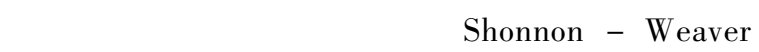

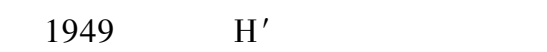

$$
\mathrm{H}^{\prime}=-\sum_{\mathrm{i}=1}^{\mathrm{s}} \mathrm{P}_{\mathrm{i}} \log _{2} \mathrm{P}_{\mathrm{i}}
$$

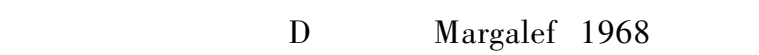
Ëã ${ }^{1} \ll \hat{E} 1 / £$

\section{$\mathrm{D} \mathbf{f}^{\prime \prime} \mathrm{S}-1 \mathrm{f} \log _{2} \mathrm{~N}$}

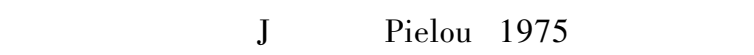
Ê1/£o

$\mathrm{J}=\mathrm{H}^{\prime} / \log _{2} \mathrm{~S}$

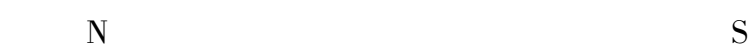

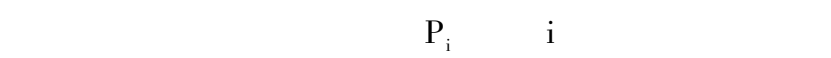

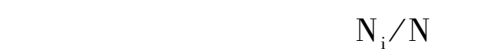

\section{3 尚 $^{1} \hat{u}$}

\section{1 ÖÖÀà \é ${ }^{3} E ́$}

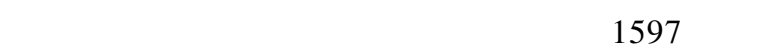

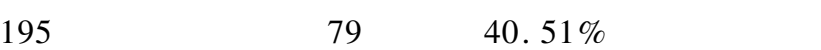

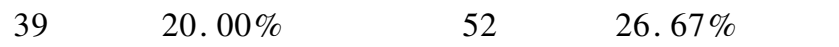

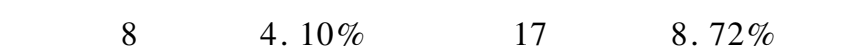

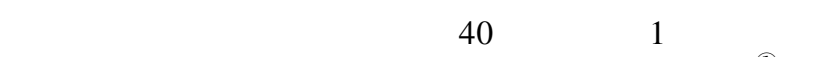

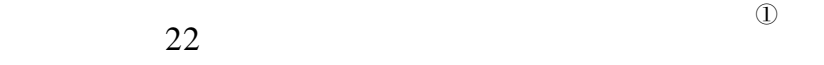

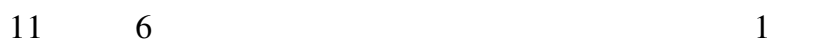

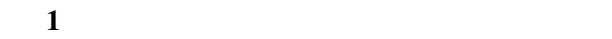

Table 1 Dominant species of macrobenthic fauna in Jiaozhou Bay

$C_{\zeta} \div \ddot{e}^{3} æ$ Sthenolepis japonica

Ėél »ëĺ »³ æAnaitides papillosa

Äâi CĐë æParalacydonia paradoxa

»", $\hat{O}^{13} \tilde{A} \ll^{3} æ S i g a m b r a$ hamaokai

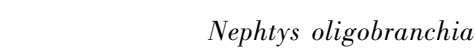

${ }^{3}$ ฉî ÇÉ 2 Ï Glycera chirori

Q̀ ×ãË $\div \mathrm{E}^{3} 2 \ddot{\mid}$ Lumbrineris heteropoda

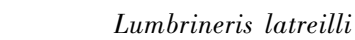

Lumbrineris longitoli

Aanides oxycephala

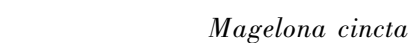

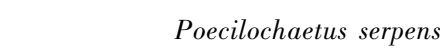

ๆàË

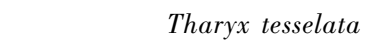

${ }^{2} \gg \mu^{1} \mid \grave{I}{ }^{3} æ$ Sternaspis scutata

$\ddot{E}_{i} \mathrm{O}$ òß/2æHeteromastus filiformis

Ф̈Đỏ3/2æ Mediomastus califoraiensis

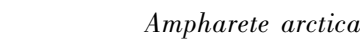

$\mu ¥ \times a ̈ E ̈ E \tilde{O} \mathfrak{Y}^{3} æ$ Amaeana antipoda

ÉáĖ̀̀ ${ }^{3}$ Terebellides stroemii

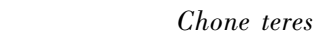

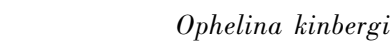

Ỡ ${ }^{2} \mathrm{O}$ ○ ĐÂÝY Eocylichna cylindrella

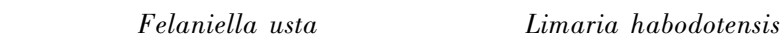

Äñð 2"î ÆE ò Raetellops pulchella

'à¿ÇÀí, ò Theora fragilis

· AEÁÉtö, òx円 Ruditapes philippinarum

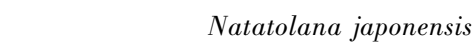

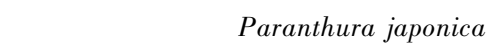

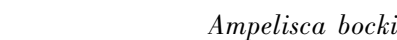

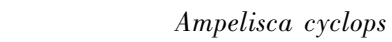

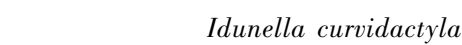

Eropisella sechellensis

İ , òǖ o Leptochela gracilis

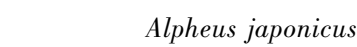

ÈpÃ«Ï, ХãĐ Raphidopus ciliatus

ఇ"

○े Хã $\mu^{1}$ ò! Đ Asthenognathus inaequipes

Çànỗ Â ýÓá Branchiostoma belcher

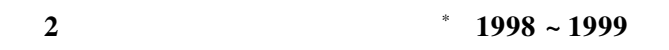

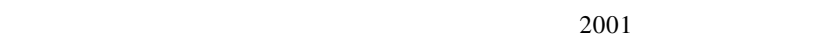

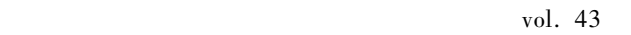


Table 2 The number of macrobenthic fauna species in samples taken from Jiaozhou Bayf 1998 - 1999 £C

\begin{tabular}{|c|c|c|c|c|c|c|c|c|c|c|c|c|}
\hline \multicolumn{3}{|l|}{$\hat{\hat{O A}}$} & \multicolumn{3}{|c|}{$\tilde{O} \tilde{\sigma} / 4$} & \multicolumn{4}{|c|}{ o̊ } & \multicolumn{2}{|r|}{$A \amalg / 2$} & \multirow{2}{*}{$\begin{array}{c}\text { XÜ } \\
\text { ÖÖẾy }\end{array}$} \\
\hline$\cdot \dot{Y}$ & 1 & 2 & 3 & 4 & 5 & 6 & 7 & 8 & 9 & 10 & 3/41 & \\
\hline $1998 / 2$ & 28 & 34 & 18 & 28 & 21 & 27 & 29 & 29 & 6 & 16 & 23.0 & 108 \\
\hline $1998 / 4$ & 31 & 29 & 28 & 24 & 23 & 35 & 41 & 9 & 9 & 28 & 24.0 & 112 \\
\hline $1998 / 8$ & 25 & 29 & 32 & 38 & 14 & 37 & 9 & 29 & 18 & 20 & 26.9 & 115 \\
\hline $1998 / 11$ & 24 & 18 & 26 & 22 & 18 & 18 & 12 & 22 & 8 & 15 & 19.0 & 84 \\
\hline $1999 / 2$ & 18 & 28 & 23 & 23 & 18 & 20 & 8 & 28 & I & 35 & 24.1 & 85 \\
\hline $1999 / 5$ & 17 & 24 & 15 & 20 & 9 & 25 & 3 & 14 & 14 & 23 & 17.9 & 81 \\
\hline $1999 / 8$ & 18 & 20 & 25 & 17 & 24 & 10 & 6 & 6 & 4 & 10 & 14.9 & 78 \\
\hline $1999 / 11$ & 14 & 12 & 26 & 21 & 21 & 16 & 7 & 13 & I & 18 & 17.6 & 76 \\
\hline
\end{tabular}

A区约 21.924.324.124.118.523.514.418.89.820.6 20.9 195

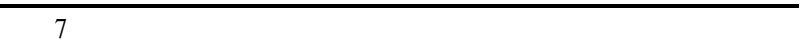

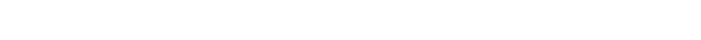

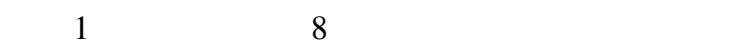

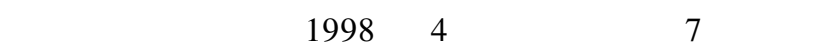

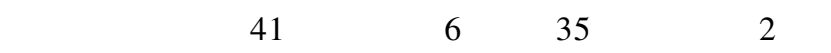

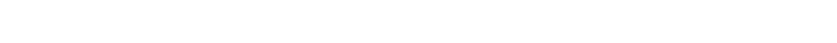

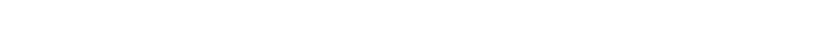

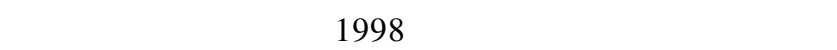

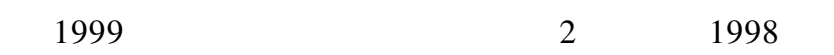

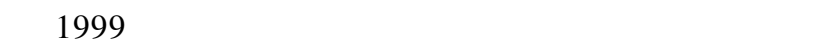

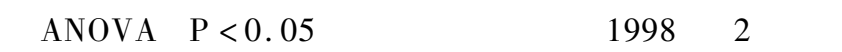

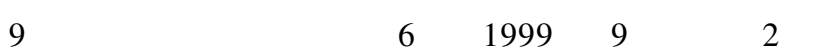

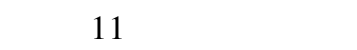

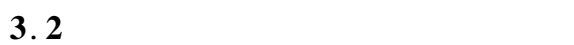

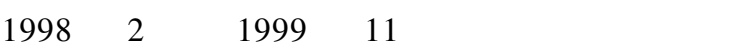

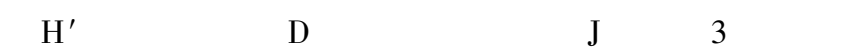

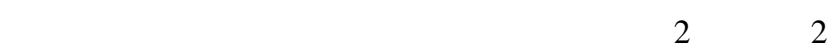

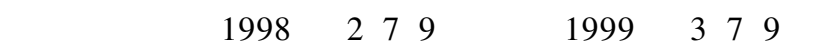

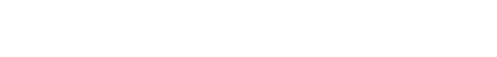

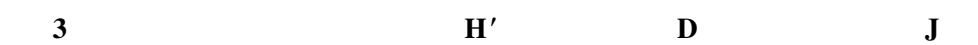

Table 3 The distribution of Shannon - Wienerf-species richness and Pielous evenness indices of macrobenthic fauna in Jiaozhou Bay from Fabruary 1998 to November 1999

\begin{tabular}{|c|c|c|c|c|c|c|c|c|c|c|c|c|}
\hline$\hat{O \hat{A}}$ & & & & & $\tilde{\sigma} \tilde{\sigma} / 4$ & & $\underline{\circ \AA}$ & & & & & $\times \hat{\cup}=10$ \\
\hline$\cdot \dot{Y}$ & & 1 & 2 & 3 & 4 & 5 & 6 & 7 & 8 & 9 & 10 & ä \\
\hline \multirow[t]{3}{*}{$1998 / 2$} & $\mathrm{H}^{\prime}$ & 4.43 & 2.13 & 3.06 & 4.08 & 4.08 & 4.28 & 4.76 & 4.23 & 2.35 & 2.14 & 4.95 \\
\hline & D & 4.80 & 4.4 & 2.86 & 4.62 & 3.77 & 4.46 & 4.78 & 4.61 & 1.22 & 2.63 & 8.83 \\
\hline & $\mathrm{J}$ & 0.92 & 0.42 & 0.73 & 0.85 & 0.93 & 0.90 & 0.98 & 0.87 & 0.91 & 0.54 & 0.73 \\
\hline \multirow[t]{3}{*}{$1998 / 4$} & $\mathrm{H}^{\prime}$ & 4.08 & 1.21 & 2.61 & 3.42 & 4.01 & 4.04 & 3.38 & 2.86 & 2.68 & 4.29 & 4.52 \\
\hline & $\mathrm{D}$ & 4.57 & 3.51 & 4.04 & 3.67 & 4.03 & 5.26 & 6.33 & 1.85 & 1.75 & 4.33 & 8.66 \\
\hline & $\mathrm{J}$ & 0.82 & 0.25 & 0.54 & 0.75 & 0.89 & 0.79 & 0.63 & 0.90 & 0.85 & 0.89 & 0.66 \\
\hline \multirow[t]{3}{*}{$1998 / 8$} & $\mathrm{H}^{\prime}$ & 4.23 & 4. 16 & 4.40 & 4.8 & 3.38 & 4.25 & 3.17 & 3.25 & 3.33 & 4.11 & 5.70 \\
\hline & $\mathrm{D}$ & 4.25 & 4.53 & 5.04 & 6.12 & 2.58 & 5.74 & 2.11 & 3.96 & 3.16 & 3.74 & 9.56 \\
\hline & $\mathrm{J}$ & 0.91 & 0.86 & 0.88 & 0.91 & 0.89 & 0.82 & 1.00 & 0.67 & 0.80 & 0.95 & 0.83 \\
\hline \multirow[t]{3}{*}{$1998 / 11$} & $\mathrm{H}^{\prime}$ & 3.01 & 1.86 & 2.89 & 3.76 & 3.86 & 4.01 & 2.77 & 4.23 & 2.79 & $3.55 \mathrm{P}$ & 4.85 \\
\hline & $\mathrm{D}$ & 3.63 & 2.47 & 4.01 & 3.86 & 3.22 & 3.44 & 2.15 & 4.02 & 1.71 & 2.95 & 7.16 \\
\hline & $\mathrm{J}$ & 0.66 & 0.45 & 0.61 & 0.84 & 0.93 & 0.96 & 0.77 & 0.95 & 0.93 & 0.91 & 0.76 \\
\hline \multirow[t]{3}{*}{$1999 / 2$} & $\mathrm{H}^{\prime}$ & 3.65 & 3.87 & 3.03 & 3.97 & 3.87 & 3.74 & 1.83 & 4.60 & I & 4.39 & 4.79 \\
\hline & $\mathrm{D}$ & 3.11 & 4.34 & 2.80 & 3.84 & 3.37 & 3.42 & 1.29 & 4.69 & / & 5.40 & 6.81 \\
\hline & $\mathrm{J}$ & 0.88 & 0.80 & 0.67 & 0.88 & 0.93 & 0.86 & 0.61 & 0.96 & / & 0.86 & 0.75 \\
\hline \multirow[t]{3}{*}{$1999 / 5$} & $\mathrm{H}^{\prime}$ & 3.67 & 3.45 & 1.29 & 3.76 & 2.74 & 4.18 & 1.49 & 3.41 & 3.48 & 3.90 & 4.65 \\
\hline & D & 3.10 & 3.88 & 1.97 & 3.47 & 1.85 & 4.11 & 0.51 & 2.61 & 2.65 & 3.81 & 6.87 \\
\hline & $\mathrm{J}$ & 0.90 & 0.75 & 0.33 & 0.87 & 0.86 & 0.90 & 0.94 & 0.90 & 0.91 & 0.86 & 0.73 \\
\hline \multirow[t]{3}{*}{$1999 / 8$} & $\mathrm{H}^{\prime}$ & 4.01 & 2.91 & 4.40 & 3.89 & 4.29 & 3.99 & 2.06 & 2.29 & 1.72 & 3.11 & 5.57 \\
\hline & $\mathrm{D}$ & 3.44 & 3.24 & 4.49 & 3.34 & 4.14 & 3.37 & 1.16 & 1.20 & 0.77 & 2.08 & 7.34 \\
\hline & $\mathrm{J}$ & 0.96 & 0.67 & 0.95 & 0.95 & 0.94 & 0.96 & 0.80 & 0.89 & 0.86 & 0.94 & 0.89 \\
\hline \multirow[t]{3}{*}{$1999 / 11$} & $\mathrm{H}^{\prime}$ & 3.50 & 3.25 & 1.72 & 3.73 & 4.23 & 3.84 & 2.50 & 3.36 & / & 3.23 & 4.66 \\
\hline & $\mathrm{D}$ & 2.47 & 2.34 & 3.75 & 3.65 & 3.94 & 3.52 & 1.41 & 2.26 & I & 3.08 & 6.82 \\
\hline & $\mathrm{J}$ & 0.92 & 0.91 & 0.37 & 0.85 & 0.96 & 0.96 & 0.89 & 0.91 & / & 0.77 & 0.75 \\
\hline
\end{tabular}

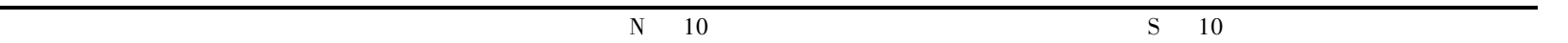

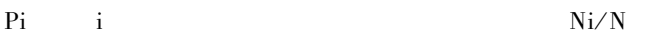




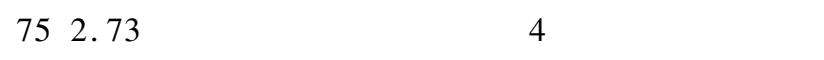

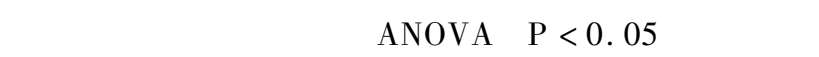

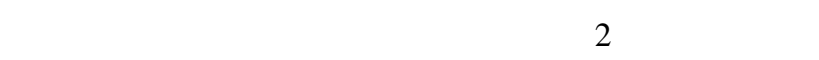

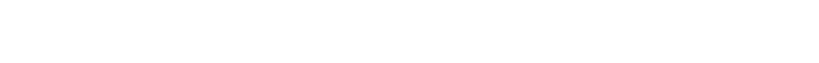

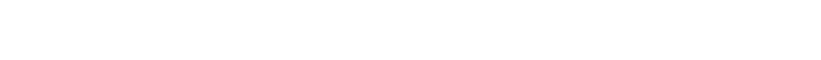

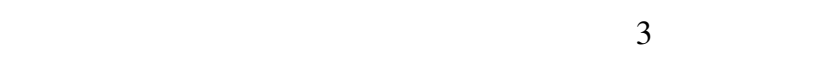

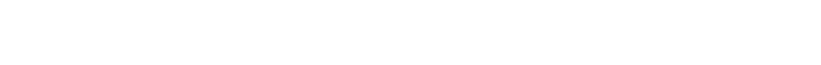

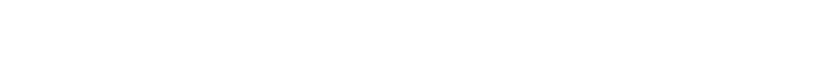

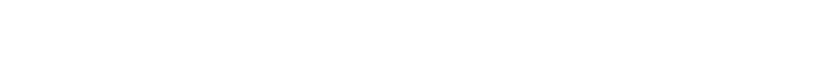

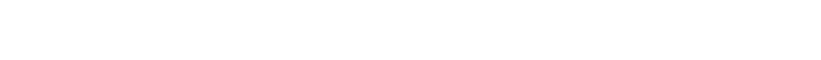

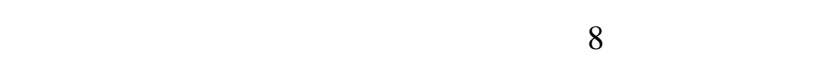

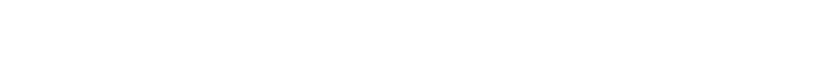

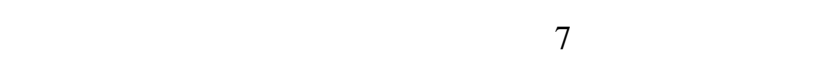

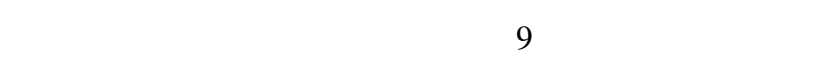

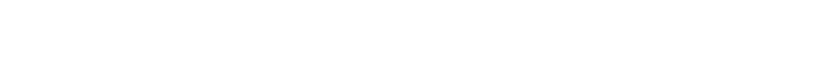

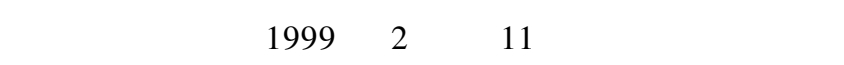

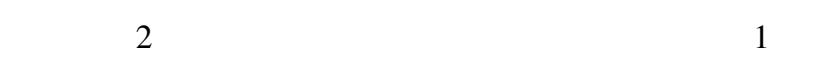

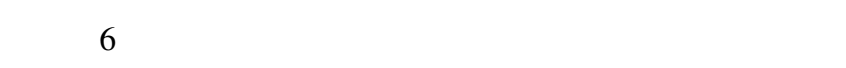

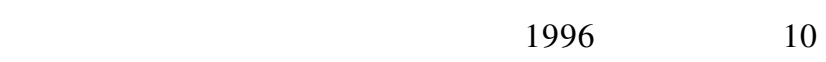

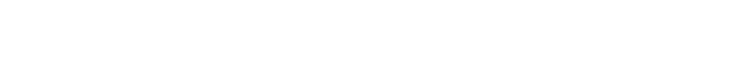

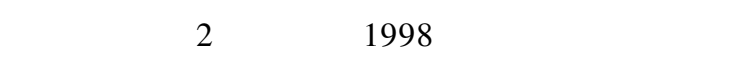

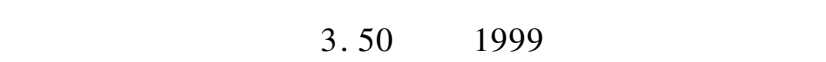

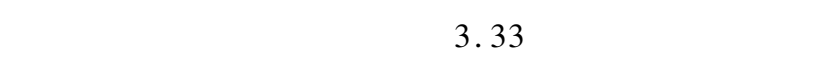

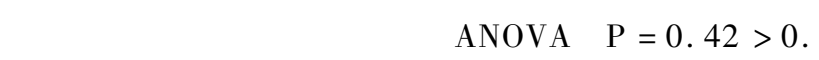
$05 £ \Subset £$

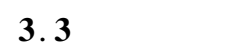

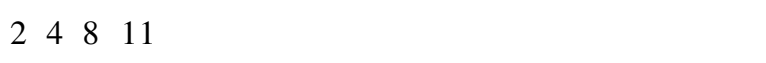

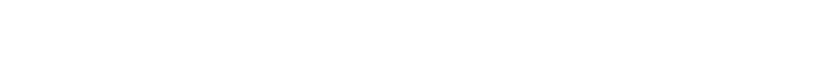

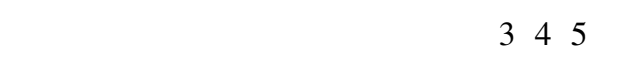

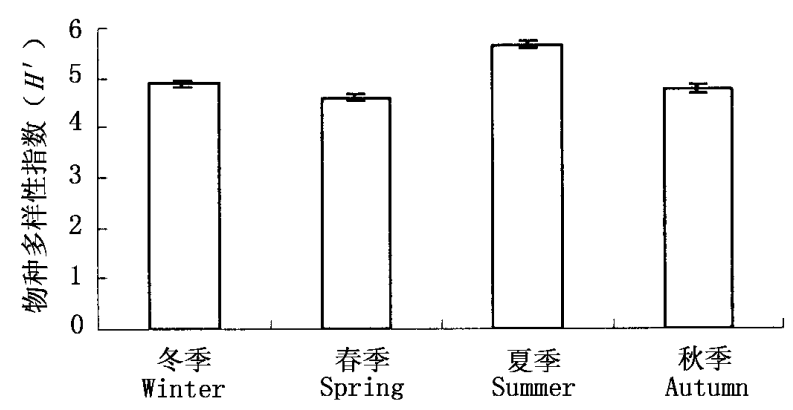

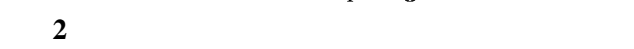

Fig. 2 Seasonal variation in Shannon - Wiener indix of macrobenthic fauna in Jiaozhou Bay

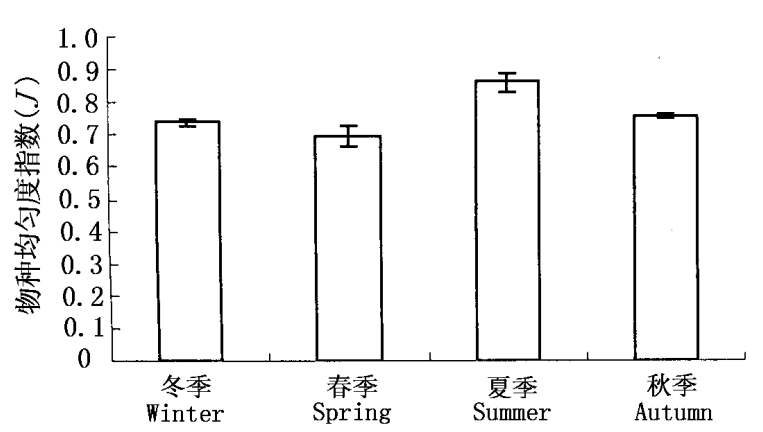

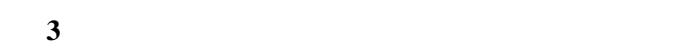

Fig. 3 Season variation in Pielous evenness indix of macrobenthic fauna of Jiaozhou Bay

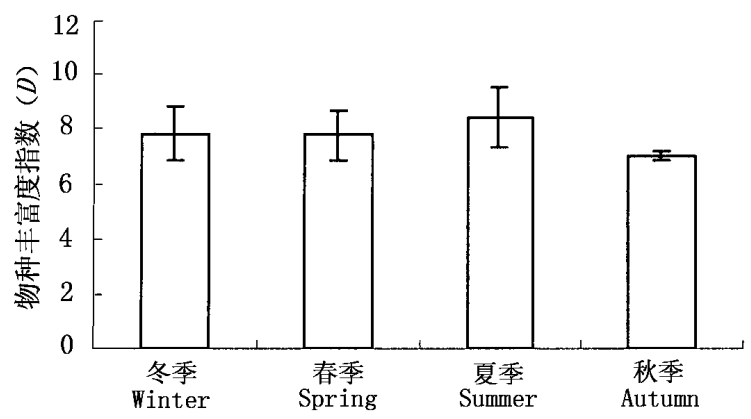

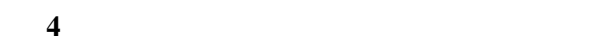

Fig. 4. Seasonal variation in species richness indix of macrobenthic fauna of Jiaozhou Bay

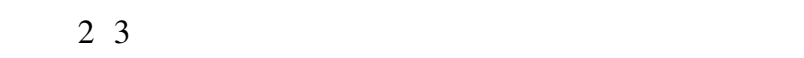

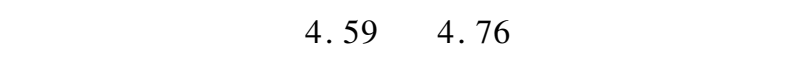

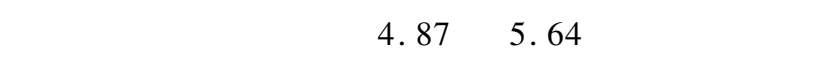

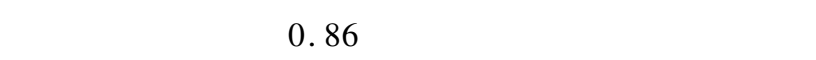

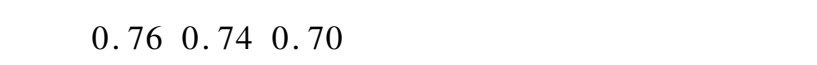

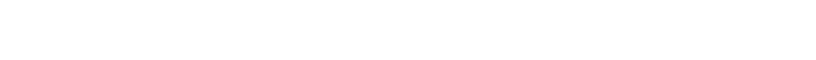

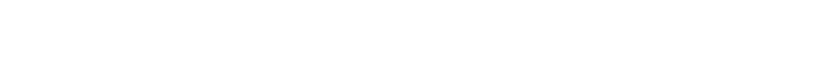

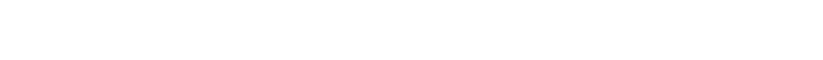

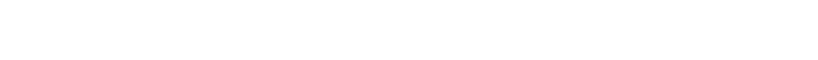

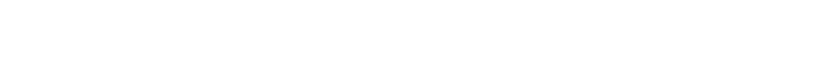

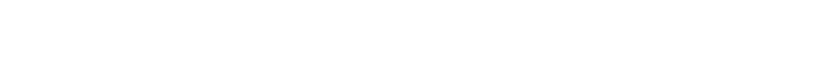

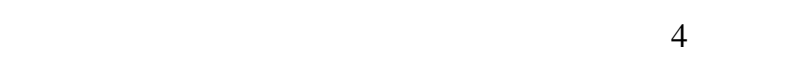
${ }^{3}$ öf

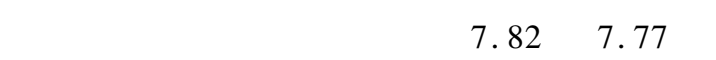

\section{4 ì ÖÂÛ}

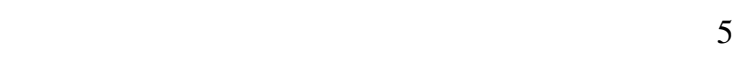

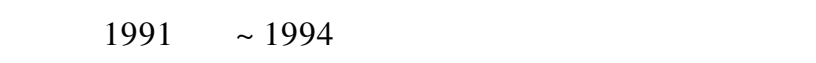

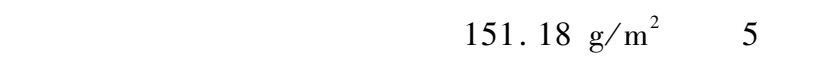

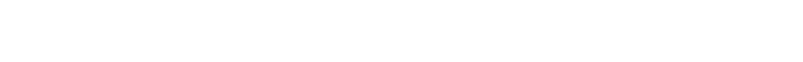

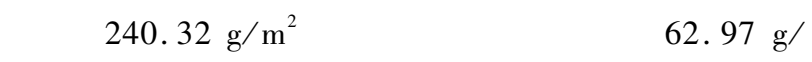




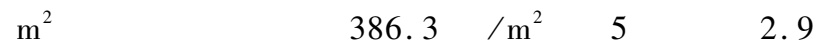

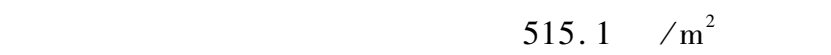

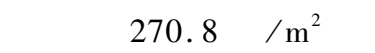

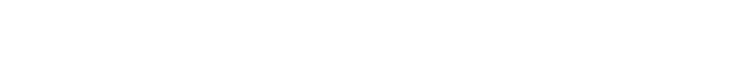

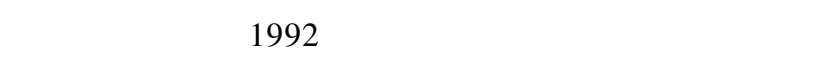

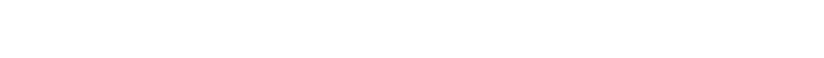

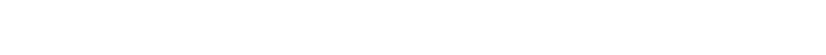

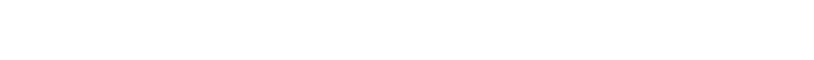
$\mu \ddot{1} 1 / \not \mu \mu_{i}$;

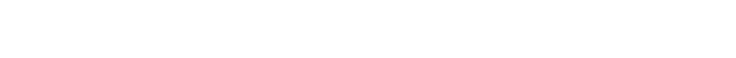

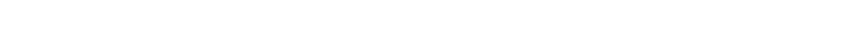

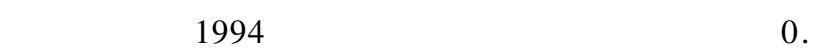

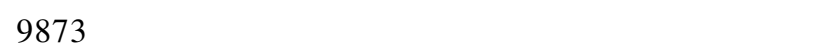
土ä» $\mu$ ÄƠỡì

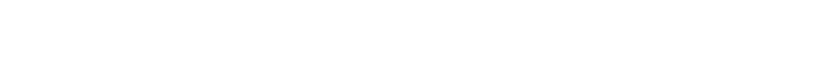

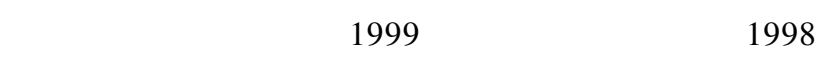

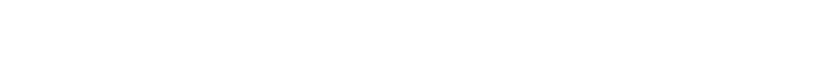

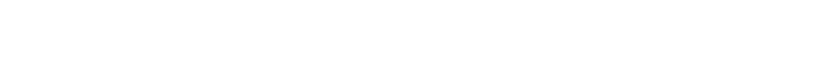

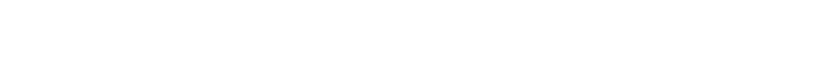
$\grave{i} \rightarrow 3 \cdot 3 \mu \ddot{A} \pm \mp \gg \alpha_{i} f$

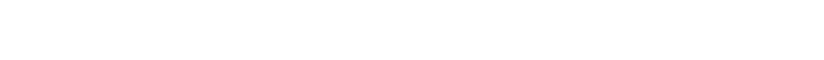

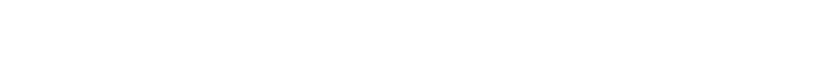

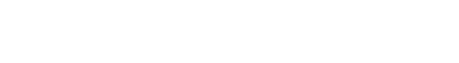

${ }^{2} \hat{\imath} \iota^{2} \hat{\wedge} \ddot{\mathrm{A}} \mathrm{A} \mathrm{x}$

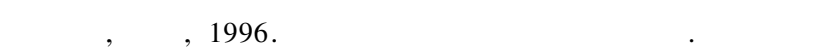
N̄, 108(6) : $58 \sim 62$

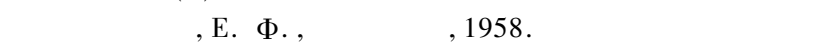

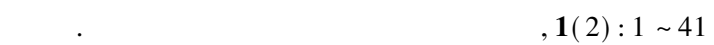

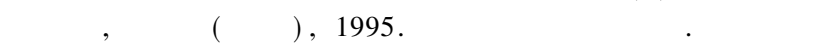

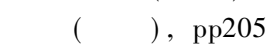

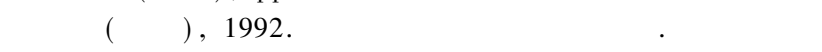
Éç $( \pm 3 / 40), p p 460$

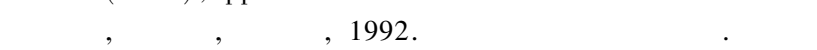

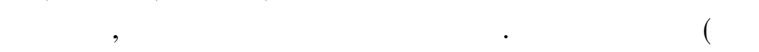
$3 / 40,220 \sim 229$

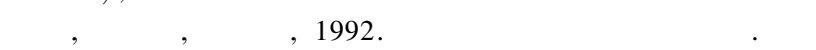

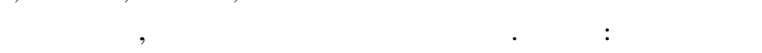
Éç, $238 \sim 256$

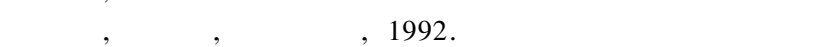

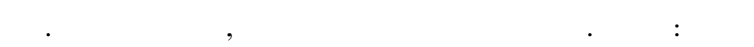

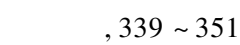

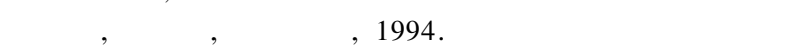

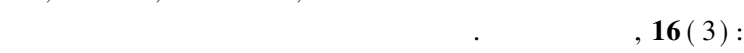
$101 \sim 112$

Margalef R, 1968. Perspective In Ecological Theory. Univ. Chicago Press, pp111

Pielou E C, 1975. Ecological Diversity, Wiley - Inters ( New York), pp163

Shannon C E and Weaver W, 1949. The Mathematical Theory of Communication. Urbanna: University of Illinois Press, pp117 